\title{
Perception of the major/minor distinction: V. Preferences among infants
}

\author{
ROBERT G. CROWDER, J. STEVEN REZNICK, and STACEY L. ROSENKRANTZ \\ Yale University, New Haven, Connecticut
}

Six-month-old infants expressed musical preferences by choosing one of two directional targets to look at, one of which produced a major chord and the other a minor chord. Over repeated testing in this way, no reliable preference for either harmony was expressed. However, a comparable choice between consonant and dissonant chords produced reliable preference for consonant harmonies.

Other things being equal, the major mode in music connotes positive emotion and the minor mode connotes negative emotion, in our culture. Arguments as to why this polarity exists (rather than the opposite connotations) remain unresolved at present (see historical reviews in Crowder, 1984, and Kastner \& Crowder, 1990). One possibility, associated with Rameau (1722/1971) and Helmholtz (1885/1954), is that the source lies with fundamental acoustic properties of periodic sounds. The opposed view is that these associations are socioculturally learned.

Looking for this particular connotation (1) crossculturally and (2) developmentally can potentially provide information relevant to this issue, even though in principle no society is so remote from Western values, and no child so young, as to have been completely untouched by the second of the two factors above. Still, both kinds of extension of the major/minor connotation render the case for (acoustic) universality more credible and the case for inculcation more farfetched. The present article describes our recent work on the developmental strategy.

Kastner and Crowder (1990) showed that children as young as 3 years old can reliably point to drawings of happy and sad faces, respectively, in response to the same tunes played in major or minor keys. This report extends that work to infants approximately 6 months of age. We used a contingent head-turning preparation (Krumhansl \& Jusczyk, 1990), because of the obvious response limitations of our subjects. The stimuli were major and minor chords. The prediction was that if the major chord connotes a positive emotion, and the minor connotes a negative one, infants would prefer the major to the minor chord.

In facing the possibility of no preference for major or minor harmonies among these infants, we decided to include a second comparison, between consonant and blatantly dissonant chords. Helmholtz (1885/1954) had asserted that the negative connotation for minor harmonies was really only a special case of the inherent distress that listeners experience for dissonance, so inclusion of this

This research was partially supported by NSF Grant GB 8608344 . Correspondence should be addressed to Robert G. Crowder, Department of Psychology, P.O. Box 11A, New Haven, CT 06520-7447. second contrast would allow observation of this distress more directly. Also, it was intended to vindicate the experimental setting with a "more dependable" comparative preference.

\section{METHOD}

\section{Subjects}

The subjects were 14 infants approximately 6 months of age (mean age $=193.38$ days, with $S D=14.71$ days, at the time of testing). Seven were males, One subject was excluded from all analyses because he never gave more than a brief glance at any of the target displays. Six of the remaining subjects received both the major/minor and consonant/dissonant sessions, in counterbalanced order. Three additional subjects received only the major/minor comparison, and 4 received only the consonant/dissonant comparison, without a second session, owing to their fussing or crying. Thus, the major/minor data were usable for 9 subjects and the consonant/ dissonant data were for 10 subjects.

\section{Apparatus and Stimuli}

The chords were produced on a Yamaha DX 100 keyboard synthesizer, using the following parameters to synthesize harmonically rich tones: Attack Rate $=19$, Decay 1 Rate $=31$, Decay 2 Rate $=0$, Decay Level $=15$, and Release Rate $=0$. The synthesizer uses an equal temperament and $A=440 \mathrm{~Hz}$. Each of the four experimental chords was played continuously and recorded for $20 \mathrm{~min}$.

The major chord consisted of the notes $\mathrm{C} 3, \mathrm{C} 4, \mathrm{E} 4$, and $\mathrm{G} 4$. The minor chord was the same, except for the lowering of the E4 to $E^{b} 4$. The consonant chord consisted of $\mathrm{C} 3, \mathrm{E} 4, \mathrm{G} 4$, and $\mathrm{C} 5$, and the dissonant chord was $\mathrm{C} 3, \mathrm{C}^{*} 4, \mathrm{~F}^{*} 4$, and $\mathrm{B} 4$.

\section{Procedure}

The subject was seated in the parent's lap, facing a panel. Mounted on the panel at eye level was a $6 \times 6$ in. wooden square with a circular pattern of light-emitting diodes. These could be made to flash on and off randomly, to create a "sparkle" effect, while a random pattern of high-pitched tones was played. This display was designed to center the infant's attention toward a hole in the center of the sparkle pattern, through which the lens of a closed-circuit TV camera was focused on the infant's face. A TV monitor was in an adjoining control room. The experimenter was in this control room, recording the infant's direction of gaze with a joystick connected to an IBM AT computer, which controlled the experiment and recorded gaze and direction times as defined by the joystick. On either side of the infant $\left(90^{\circ}\right.$ relative to straight ahead), just above the infant's eye level, were loudspeakers with boxes on top, each of which housed a toy bear holding a toy megaphone. The boxes were covered with dark Plexiglas, so that the bears could be seen only when the lights inside the boxes were illuminated.

Each trial began when the experimenter turned on the "sparkler" to center the infant's attention. Once the infant looked directly at the spar- 
kler, it was extinguished, and the light in the box either to the left or the right was illuminated, so that the corresponding bear became visible. When the infant looked at either box, a chord was played through the speaker located on that side. This chord remained on until the infant looked away for 2 consecutive seconds, or until $45 \mathrm{sec}$ had elapsed. This event marked the end of that trial. Each session included 8 training trials (to familiarize the infant with this procedure and to allow learning of which chord went with which side) and 12 identical test trials following immediately after practice.

Six infants participated in two sessions, separated by a 5 -min break with a parent in the adjoining waiting room. In counterbalanced order, one session contained the major/minor distinction, and the other contained the consonant/dissonant distinction. Seven received only one of these two distinctions, as noted above. The major and minor (or consonant and dissonant) chords were assigned to one side and remained located there for a particular subject, within a session, but across subjects this assignment was counterbalanced. The major and consonant chords, which were very similar, were assigned to opposite sides for a given child. Throughout the experiment, the parent listened to extraneous music presented over headphones, in order to mask the auditory stimuli being presented to the infant. The parent observed the child's face on a small monitor above and behind the apparatus, out of the child's field of vision. This ensured that the child's face was in position for gaze coding and also prevented the parent from observing the illumination of the bears.

\section{RESULTS}

The mean number of seconds for looking at the bear associated with the major chord was $2.67(S D=1.52)$, whereas the mean time for looking at the source of the minor chord was $2.60 \mathrm{sec}(S D=1.61)$. These mean gaze times were not reliably different $[t(8)=0.1]$. For the consonant chord, the mean time was $2.85 \sec (S D=1.39)$, and for the dissonant chord, it was $1.98 \mathrm{sec}(S D=1.35)$. There was a reliable preference for the consonant over the dissonant chord $[t(9)=2.43, p<.025]$.

Of the 9 subjects tested on the major/minor distinction, 5 looked longer at the major side and 4 at the minor side. Of the 10 tested on the other distinction, 8 looked longer at the consonant side and 2 at the dissonant $(p=.055$, by a sign test).

Inclusion of the training trials as test trials rendered both of the distinctions nonsignificant, so we remained with our originally planned choice of the target test trials.

\section{DISCUSSION}

The absence of a major/minor preference in looking time was unexpected in this experiment and thus reassures us that experimenter bias did not guide our results. Nor can the negative result be ascribed to an insensitive experimental technique, because a reliable preference was shown in the consonant/dissonant comparison.

We note that our procedure was adopted after a number of false starts, in which we tried such protocols as free operant choice, using headturning, and manual responses. We were unable to surmount strong asymmetrical preferences with these techniques and decided on the present method after reading of a comparable study, with what we considered even more subtle musical distinction, by Krumhansl and Jusczyk (1990).

Of course, one possibility is that the major/minor distinction, as realized in these synthetic chords, was still too subtle to be differentially pleasing to 6-month-old infants. The results for the (to us) more blatant consonant/dissonant distinction are consistent with the claim that the latter distinction was more salient than the former.

A second possibility is that the major/minor connotations are socially transmitted, after all, and that the timing of this learning is somewhere between 6 months and Kastner and Crowder's (1990) 3 years. For this second interpretation, the results with the consonant/dissonant distinction are something of an embarrassment: It must then be explained how these young infants had time to learn the consonant/dissonant connotation but not the major/minor connotation. Alternatively, one could claim that the consonant/dissonant distinction is inherently acoustical in source, but that the major/minor distinction is not. Awaiting more evidence, we cannot argue strongly for either position, although explaining why one of these distinctions should be inherently acoustical and the other altogether learned seems a stiff assignment.

\section{REFERENCES}

Crowder, R. G. (1984). Perception of the major/minor distinction: I. Historical and theoretical foundations. Psychomusicology, 4, 3-10.

Helmholtz, H. L. F. (1954). On the sensations of tone (4th ed.; A. J. Ellis, Trans.). New York: Dover. (Original work published 1885)

Kastner, M. P., Crowder, R. G. (1990). Perception of the major/minor distinction: IV. Emotional connotations in young children. Music Perception, 8, 189-202.

KrumhansL, C. L., JusczyK, P. W. (1990). Infants' perception of phrase structure in music. Music Perception, 1, 70-73.

RameaU, J. (1971). Treatise on harmony (P. Gossett, Trans.). New York: Dover. (Original work published 1722)

(Manuscript received September 8, 1990.) 